\title{
Thermal comfort evaluation of the enclosed transitional space in tropical buildings: Subjective response and computational fluid dynamics simulation
}

\begin{abstract}
This study aims to identify the thermal environment of an enclosed transitional space in a tropical educational institution and occupants' perceptions on thermal comfort. The methods applied were field survey, which covered objective measurement and subjective assessment, as well as Computational Fluid Dynamics (CFD)simulations. Comparisons were made between the empirical and predicted results. In this study, it was identified in the field assessment that most of the respondents were satisfied with the thermal environment in the enclosed lift lobby and preference was directed towards cooler environment. The predicted results showed fair agreement with the empirical results, with minor differences between the two results for the thermal and airflow conditions. Besides, a lower expectation factor in PMV index is required for thermal environment in transitional spaces. Analysis of thermal neutrality in this survey has demonstrated that the thermal conditions in the enclosed lift lobby were acceptable by $80 \%$ of the respondents. The methods applied in this study can be extended to other enclosed transitional spaces in the tropical buildings.
\end{abstract}

Keyword: CFD; Educational institution; Lift lobby; Thermal comfort; Transitional space; Tropics. 